\title{
The complex ontogenetic trajectory of mandibular shape in a laboratory mouse
}

\author{
Donald L. Swiderski ${ }^{1}$ and Miriam L. Zelditch ${ }^{2}$ \\ ${ }^{1}$ Kresge Hearing Research Institute and Museum of Zoology, University of Michigan, Ann Arbor, MI, USA \\ ${ }^{2}$ Museum of Paleontology, University of Michigan, Ann Arbor, MI, USA
}

\begin{abstract}
The mouse mandible is a popular model system that continues to be the focus of studies in evo-devo and other fields. Yet, little attention has been given to the role of postnatal growth in producing the adult form. Using cleared and stained specimens, we describe the timing of tooth and jaw development and changes in jaw size and shape from postnatal day 1 ( $p 1$ ) through weaning to adulthood. We found that tooth development is relatively advanced at birth, and that the functional adult dentition is in place by p15 (just before the start of weaning). Shape analysis showed that the trajectory of mandible shape changes direction at least twice between birth and adulthood, at p7 and p15. At each stage there are changes in shape to all tooth- and muscle-bearing regions and, at each change of direction, all of these regions change their pattern of growth. The timing of the changes in direction in Mus suggests there are signals that redirect growth patterns independently of changes in function and loading associated with weaning and jaw muscle growth. A better understanding of these signals and how they produce a functionally integrated mandible may help explain the mechanisms guiding evolutionary trends and patterns of plasticity and may also provide valuable clues to therapeutic manipulation of growth to alleviate the consequences of trauma or disease.
\end{abstract}

Key words: mandible; Mus; ontogeny; postnatal; shape.

\section{Introduction}

The newly formed mouse mandible is quite different in size and shape from the adult structure. All the components of the mature mandible are present by embryonic day 17 (3-4 days before birth), when the expanding sheet of membrane bone has completed its envelopment of Meckel's cartilage, the dental primordia and the cartilages that will form the posterior processes (Ishizeki et al. 1999; Ramaesh \& Bard, 2003). But at this age, the posterior processes are relatively shorter than in the adult and are little more than cartilaginous rods ensheathed in bone where they join the horizontal ramus. The vertical plates that will provide structural support for the posterior processes have just begun to fill the spaces between them. Thus, the conformation of the newly formed mandible is quite different from that of the adult bone.

The substantial difference in shape between embryonic and adult mandibles leads to the question of what path the

\section{Correspondence}

Donald L. Swiderski, Kresge Hearing Research Institute and Museum of Zoology, University of Michigan, Ann Arbor, MI, USA.

E: dlswider@umich.edu

Accepted for publication 3 September 2013

Article published online 22 September 2013 ontogenetic trajectory of the bone takes through shape space. More specifically, is the path straight or not and, if it is not straight, does it follow a mathematically simple curve (like a ballistic trajectory or a log spiral) or a more complex route that cannot be described by a simple formula? The answer to this question could have several important implications. The functional consequences of mandibular shape for feeding performance suggest that the ontogenetic sequence of shapes may influence an immature individual's ability to progress from nursing to processing foods typically eaten by adults (Biknevicius, 1996; Tanner et al. 2010; La Croix et al. 2011). Consequently, variation in the ontogenetic trajectory of shape could affect fitness of both the offspring and parents. Variation in the trajectory can affect the offspring's ability to grow and mature, as well as produce deviations from the appropriate adult shape. Variation in the trajectory can affect the parent's fitness by its consequences for current reproductive effort and parental survival plus success of subsequent litters. In addition, the organization of variation in the ontogenetic trajectory (coordinated deviations or correlated downstream effects) may have implications for the potential evolutionary transformations of the trajectory and the target adult shape (Zelditch et al. 2009).

Although the mouse mandible has become an important model system in studies of development, evolution and medicine, the progressive transformation of the 
newly formed mandible to the adult size and shape has not yet been described in detail. Numerous studies have analyzed covariance patterns in adults (Atchley et al. 1985; Bailey, 1985; Cheverud et al. 1997; Klingenberg et al. 2003, 2004). Many others describe differences in adult form resulting from genetic divergence of laboratory strains or natural populations (Renaud et al. 2009; Sans-Fuentes et al. 2009; Boell et al. 2011; Muñoz-Muñoz et al. 2011; Burgio et al. 2012; Siahsarvie et al. 2012). There also are several analyses of deviations from normal adult form caused by diet manipulations and mutations that directly or indirectly affect bone growth (Lightfoot \& German, 1998; Fujita et al. 2004; Ramirez-Yañez et al. 2005; Rot-Nikcevic et al. 2007; Tsutsui et al. 2008; Renaud et al. 2010). Many other studies have identified genes contributing to proper formation of the embryonic mandible (Depew et al. 1999; Ruest et al. 2004; Dixon et al. 2006; Shibata et al. 2006; Oka et al. 2007; Anthwal et al. 2008; Sun et al. 2012). But despite this voluminous literature, there do not appear to be any studies that explicitly examine the ontogeny of mandibular shapes from neonatal to adult. A better understanding of this important transformation could serve as a starting point for investigating both evolutionary transformations of growth trajectories and potential therapies for abnormal growth.

The focus of this study is to describe the postnatal trajectory of jaw shape in a commonly used inbred strain of laboratory mice: C57BL/6J. To provide a temporal and biological framework for this analysis, we first describe the timing of major developmental milestones in mandibular development (e.g. ages of tooth eruption and weaning). Then, using geometric morphometrics, we describe changes in jaw shape from postnatal day 1 ( $p 1)$, through several closely spaced postnatal ages to a few days after weaning, evaluating the progress at those ages toward the adult shape, which is represented by a sample of 3-month-old individuals. We analyze the correlation of shape with mandibular size during postnatal growth and also evaluate the deviations of the jaw shape ontogeny from a linear trajectory. Our goal is to provide a basis of comparison for studies of variation in ontogenetic patterns in Mus as well as in rodents in general.

\section{Methods}

Specimens used in this study were C57BL/6J mice raised for an unrelated project. All animals were housed and euthanized following standard protocols approved by the University Committee on the Use of Care of Animals of the University of Michigan.

\section{Specimen preparation and photography}

Specimens were collected at 2-day intervals during the first postnatal week, then at 3-6-day intervals until p24 (approximately 3 days after weaning). Adult size and shape are represented by individuals at 86 days. Sample sizes were three to five at each time point, for a total of 31 individuals.
One obstacle to quantifying early transformations of mandibular morphology is its lack of ossification. Not only do the posterior processes (coronoid, condylar and angular) terminate in large cartilages; most of the bone that has formed is woven. Both tissues are quite vulnerable to mechanical damage and are difficult to image radiographically. To circumvent these problems, mandibles collected between birth and weaning were cleared and stained following a protocol modified from McLeod (1980). This technique stains cartilage blue (Alcian blue) and bone red (Alizarin), and renders most other tissues transparent. The primary changes to McLeod's protocol were to decapitate animals immediately after anesthetic overdosing, and to skin the heads to allow more rapid diffusion. In addition, the amounts of time specimens were placed in each solution were extended as needed to stain and then clear the tissues of progressively larger specimens.

After clearing and staining, jaws were then dissected away from the skull, and soft tissue was removed from the mandible so that it could be placed in a standard orientation and photographed with minimal optical distortion. To further reduce distortion, all specimens were photographed while completely immersed in glycerin. Photographs were taken with an Insight QE 3-shot color digital camera mounted on a Leica MZ12 dissecting scope and captured using SPOT image analysis software (version 4.6, Diagnostic Instruments). The Sрот software also was used to embed a scale bar in each image for size calibration.

\section{Digitizing and superimposition}

Mandibular size and shape were computed from the coordinates of 60 points (Fig. 1). Fourteen of the points are anatomically distinct loci (landmarks sensu Bookstein, 1991), including openings of tooth alveoli, corners or tips of processes, and locations where processes connected to the ramus or the sheets of bone between processes. The remaining 46 points are evenly spaced along curves between landmarks (semilandmarks; Bookstein, 1997a,b), on curves outlining the posterior processes (coronoid, condylar and angular), on the diastema (dorsal margin of the horizontal ramus between incisor and molar), and along the ventral margin of the horizontal ramus. For each curve, the same number of points was recorded on every specimen; therefore, to minimize effects of digitizing error, only five to seven points were used on the curves on the posterior processes, which are quite short at young ages. Larger numbers of semilandmarks were digitized on the longer curves on the diastema (9) and ventral edge of the ramus (13). As our measure of size, we use centroid size (square root of the summed squared distances of

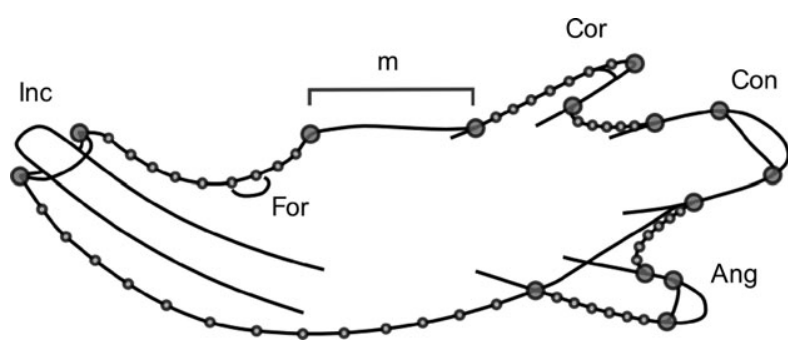

Fig. 1 Digitized points on the outline of a mandible at postnatal day 1. Large dark circles are landmarks; smaller lighter circles are semilandmarks. Anatomical features indicated are: molar alveolus (m, bracket), coronoid process (cor), condylar process (con), angular process (ang), mental foramen (for), and incisor (inc). 
points from their centroid; Bookstein, 1991), computed from all 60 points for each specimen.

Shapes were aligned by Procrustes superimposition to remove differences in location, orientation (rotation) in the photographic plane, and scale. The coordinates of the semilandmarks contain an additional nuisance parameter (position along the curve), which is removed by sliding them to minimize bending energy, a measure of local deformation (Green, 1996; Bookstein, 1997a). Differences between two superimposed shapes are given as their Procrustes distance, a function of the summed squared differences between coordinates of corresponding points. Further mathematical details can be found in Marcus et al. (1996) and Zelditch et al. (2012), in the references cited in these works, and in the help files of the programs used to perform the computations (below).

Coordinates of landmarks and semilandmarks and the length of the scale bar were recorded using TPSDIG, versions 1.4 and 2.10. Procrustes superimposition (including semilandmark sliding to minimize bending energy) and computation of centroid size was performed in TPSRELW. Both programs are available from F. J. Rohlf at http://life.bio.sunysb.edu/morph.

\section{Analysis}

Change in mandibular size was evaluated by fitting a series of growth models (Chapman-Richards, Logistic, Monomolecular, two forms of Gompertz, Von Bertalanffy, Quadratic, and Linear) to the data for mandibular centroid size. The same models were fit to a measure of shape maturity, the Procrustes distances between the mean shape for each age and the average shape at the youngest age (Zelditch et al. 2003). For both size and shape, models were first assessed by testing for significant autocorrelations among residuals (i.e. systematic age-dependent deviations from expected values) by bootstrapping. If the autocorrelation of residuals seen in the original data exceed those in $95 \%$ of 400 replicates, the autocorrelation of the residuals was deemed to be significantly greater than expected by chance, and the model was excluded from subsequent analyses (Zelditch et al. 2003). To determine which model fits best, we used the Akaike information criterion (AIC). This metric and the variance explained were also used to rank models when none of them fit well. Model details are available in Zelditch et al. (2003).

Because both size and shape can be linear relative to each other when both follow the same non-linear model, we tested for a linear relationship between shape and size by regressing shape on centroid size. After computing the direction through shape space that is maximally correlated with variation in size, the data were projected onto the regression vector to obtain scores representing their expected values. Additionally, principal components analysis (PCA) was used to visualize the major dimensions of variation, with scores on those axes providing a preliminary check for changes in the direction of shape change. Based on the PCA, which indicated that there are changes in the orientation of the trajectory, and life history, three intervals were delimited. To determine whether the ontogenetic trajectory changes direction by more than expected by chance, we measured the angle between the two phases. The angle between the trajectories is a common measure of the difference in orientation of two vectors (Zelditch et al. 2000); when the two vectors are in the same direction, the angle between them is zero, so the statistical analysis determines whether the observed angle exceeds that expected by chance. The test is done by first regressing shape on size, then randomly drawing two samples of residuals, with replacement, from each original sample (here, interval). For each of the two intervals, the residuals are added to the expected values for their size, and the angle is computed between the two vectors, a process that was iterated 400 times. This gives the distribution of angles that can be obtained by resampling within each interval. If the angle between the two original trajectories exceeds the angles between $95 \%$ of the resampling sets for both intervals, the angle between trajectories can be considered statistically significant and the directions can be considered significantly different.

Several analyses were performed using programs in the IMP series, which are available from H.D. Sheets at http://www3.canisius. edu/ sheets/morphsoft.html. Growth curves were estimated by fitting the observed sizes or shape distances to published growth models using GrowChoice. PCA, including Anderson's test for the number of distinct eigenvalues, was performed using PCAGEN7a. The test for a significant difference between directions of two ontogenetic shape trajectories was performed using VECCOMPARE7. Illustrations of differences between two shapes (e.g. mean shapes at two ages) were produced in TwoGRoup7. Regression of shape on size was performed both in the IMP program REGRESS7 and in MORPHOJ (available from C. P. Klingenberg at http://www.flywings.org.uk/ MorphoJ). REGRESS7 was used to compute the proportion of shape variance explained by the regression, and Goodall's F; it was also used to perform the permutation test for statistical significance. MORPHOJ was used to compute the scores of individual specimens on the regression vector.

\section{Results}

\section{Relative timing of tooth and jaw development}

Qualitative examination of cleared and stained specimens at $\mathrm{p} 1$ shows that the mandible has the general outline and all the basic components of a rodent jaw (Fig. 2). Alizarin red staining reveals ossified membrane enveloping the tooth primordia and merging with the bases of the three posterior processes (coronoid, condylar and angular), which already are prominent and easily recognized. Alcian blue staining shows that each is capped with a thick cartilage and that a remnant of Meckel's cartilage emerges from the horizontal ramus between the condylar and angular processes. Meckel's cartilage is still continuous with the precursors of the middle ear bones and persists through at least p3, but is gone by $p 5$.

The lateral view of the $\mathrm{p} 1$ mandible also reveals a void where the first molar (m1) is developing but not readily visible. Below this void, the masseteric ridge can also be identified as a relatively dense region of bone extending toward the angular process. A second void occupied by the incisor root also can be seen in the ventral part of the horizontal ramus; it does not yet extend beyond the molar primordium. The incisor itself is visible near the alveolar opening. Although the incisor protrudes through the alveolar opening, it has not yet emerged through the oral epithelium.

The crown of $\mathrm{m} 1$ is more clearly seen in lateral view at p3, and the alveolar void is visibly expanded to make room for $\mathrm{m} 2$. By $\mathrm{p} 5$, the crown of $\mathrm{m} 2$ also can be seen and the incisor alveolus now extends beyond $\mathrm{m} 2$. By $\mathrm{p} 7$, the crown of $\mathrm{m} 1$ appears to be nearly complete. Throughout this 
Fig. 2 Mandibles in lateral view at postnatal days $1,3,7,10,18$ and 24 . Specimens were cleared and stained to show bone (red) and cartilage (blue). Because Alcian blue labels collagen, residual periosteal membrane and epithelial tissue also are labeled. Scale bar: mm. MC, Meckel's cartilage; m1, first molar; m2, second molar.

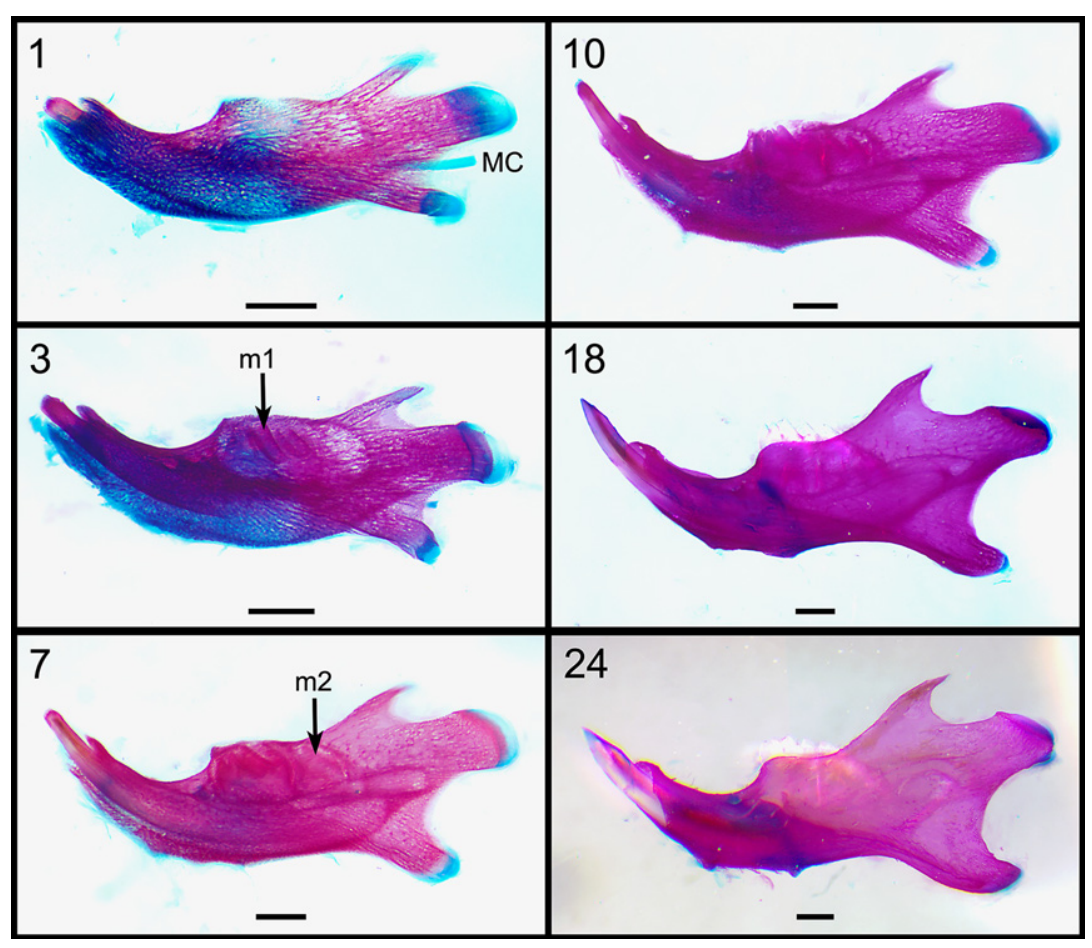

period, both $\mathrm{m} 1$ and $\mathrm{m} 2$ often are visible in dorsal view because the bone frequently does not grow completely over the occlusal surface before eruption begins; consequently, protrusion of $\mathrm{m} 1$ at this age may reflect that lack of bone growth rather than active extrusion of the tooth. In some p7 individuals, the length of incisor extending out of the alveolus is noticeably greater than in younger specimens, suggesting that incisor extrusion may have begun in those individuals. Molar eruption is evident by p10, but their roots have just begun to form. By $\mathrm{p} 15, \mathrm{~m} 3$ can be seen through the bone, and by p18 it may begin to emerge through the alveolar bone. After p15, if not earlier, further elongation of the mandible is not needed to accommodate growth of the molars. The incisor root does continue to grow beyond the posterior end of the molar row, but the space it requires has already been provided by earlier growth of the condylar process.

We observed evidence of wear on the incisor and $\mathrm{m} 1$ of one animal at p15 and on $\mathrm{m} 2$ in all our p18 animals. In contrast, $\mathrm{m} 3$ had not reached the occlusal plane and did not exhibit wear even at p24. Because $\mathrm{m} 3$ is much smaller than the other teeth, may not reach the occlusal plane until the other teeth are substantially worn, and accounts for only about $15 \%$ of the adult molar crown area, it can be considered rudimentary. Thus, these mice have the complete effective adult dentition in place with the eruption of $\mathrm{m} 2$ at the beginning of weaning.

As the teeth are developing, the shape of the jaw also undergoes dramatic changes in size and shape. These changes are described quantitatively in the next sections; here we note some qualitative changes that are not immediately obvious in the quantitative data. One set of those concerns the relative sizes of the cartilages at the tips of the posterior processes. As mentioned above, the membrane bone enveloping the tooth primordia has already merged with the ossified bases of the posterior processes at $\mathrm{p} 1$. Approximately the distal third of these processes remains cartilaginous, although the lower half of that appears to be ringed by bone in the two larger processes (the angular and condylar). The relative sizes of these cartilage caps diminish rapidly as the processes elongate, but their absolute sizes diminish much more slowly. The tip of each process remains cartilaginous as late as p24.

As the posterior processes elongate, they also change shape and orientation. As these changes take place, the positions of the anterior edges of the coronoid and angular processes remain fairly stable near the posterior end of the developing molar row. As the processes shift posteriorly, they also tilt forward over time, increasing the angle between them; and the tips of both processes hook more posteriorly with age. As the processes elongate, the sheets of bone connecting their bases also expand along the lengths of the processes, moving progressively closer to the tips as the cartilages become relatively smaller.

\section{Growth models}

All growth models produced residuals with significant autocorrelation when fit to the size data (Table 1A). Thus, none of the models can be considered to fit the data well. Even 
Table 1 Fit of growth models to mandibular size and shape.

\begin{tabular}{ccccccc}
\hline Model & Autocorr & Exp & AIC & Wt & $\%$ var & Age $@ 95 \%$ \\
\hline \multicolumn{7}{l}{ (A) centroid size } \\
MM & 0.389 & 0.353 & 12.01 & 0.201 & 98.3 & 28 \\
vB & 0.415 & 0.259 & 12.11 & 0.191 & 98.1 & 26 \\
G1 & 0.433 & 0.342 & 12.20 & 0.183 & 98.0 & 25 \\
og & 0.489 & 0.290 & 12.53 & 0.156 & 97.7 & 23 \\
G2 & 0.563 & 0.330 & 13.19 & 0.112 & 96.8 & 25 \\
CR & 0.386 & 0.199 & 14.01 & 0.074 & 98.2 & 28 \\
Qd & 0.739 & 0.341 & 14.35 & 0.063 & 94.2 & na \\
Lin & 0.874 & 0.372 & 16.59 & 0.020 & 51.4 & na \\
(B) Procrustes distance from shape at p1 & & \\
MM & $0.205^{*}$ & 0.346 & -4.78 & 0.207 & 97.6 & 35 \\
vB & $0.295^{*}$ & 0.339 & -4.54 & 0.184 & 97.3 & 30 \\
G1 & 0.357 & 0.333 & -4.32 & 0.165 & 96.9 & 28 \\
Log & 0.496 & 0.336 & -3.72 & 0.122 & 95.9 & 24 \\
G2 & 0.535 & 0.328 & -2.91 & 0.081 & 93.8 & 28 \\
CR & $0.208 *$ & 0.346 & -2.78 & 0.076 & 97.6 & 35 \\
Qd & 0.534 & 0.288 & -3.93 & 0.135 & 96.3 & na \\
Lin & 0.836 & 0.315 & -0.90 & 0.030 & 53.9 & na \\
\hline
\end{tabular}

MM, monomolecular; vB, von Bertalanffy; G1, Gompertz as implemented by Fiorello \& German (1997); Log, logistic; G2, Gompertz as implemented by Zullinger et al. (1984); CR, Chapman-Richards; Qd, quadratic; Lin, linear regression; autocorr, observed autocorrelation; exp, expected autocorrelation; AIC, Akaike information criterion; wt, AIC weight; \%var, proportion of variation described by the model; Age@95\%, age at which the variable is predicted to reach $95 \%$ of the asymptotic value. *Autocorrelation is not significant.

All models have significant autocorrelation.

so, all of the sigmoidal models explained at least $96 \%$ of the variation, which was better than the quadratic or linear models. The monomolecular model explained the highest proportion of the observed variation and had the highest AIC weight. When the growth models were fit to the shape data (Procrustes distances from average shape at p1), most of the models again produced residuals with significant autocorrelation (Table 1B). The exceptions were the monomolecular, Chapman-Richards and von Bertalanffy models. Again, the monomolecular model explained the highest proportion of the observed variation and also had the highest AIC weight.

As Figure 3A shows, the monomolecular model passed through the scatter of observed size values at every age but p18. At this age, observed values were well below the value predicted by the model, and not much higher than the values observed at p15. At p18, the model predicts jaw size will be at $90 \%$ of the asymptote and by p24, it will reach $95 \%$ of the asymptote, and this expectation was met by that sample. This model also estimated that onset of growth $\left(T_{0}\right)$ was 6 days before birth when osteoid formation has just begun. The monomolecular model for shape maturation also predicted the observed values well at almost every age (Fig. 3b). This is somewhat more remarkable than the fit to the size data because there are two intervals during which there appears to be little if any shape change ( $p 3-p 5$ and p7-p10). For each of these two intervals, the curve of predicted values passes through the lower end of the range at the younger age and then through or near the upper end of the range at the older age. Interestingly, the inferred onset of shape change was just 1 day before birth, several days later than the onset of size change.

Comparison of the growth curves for jaw size and shape suggests that shape matures later than size. Shape begins to change closer to birth, and at every subsequent milestone, shape is predicted to be relatively farther from its adult asymptote than is size, meaning that shape is consistently less mature than size. In addition, shape is expected to reach $90 \%$ of the asymptotic value at p27 and $95 \%$ at p35, which are 9 and 11 days after size is expected to reach the corresponding marks. Thus, shape maturity may lag slightly behind size, but both size and shape are close to or even overlap adult values within a few days of weaning.

\section{Correlation of shape and size}

The similarity of the best fitting growth curves suggests a high correlation between jaw size and shape during postnatal growth. This inference was strongly supported by regressing shape on centroid size. Size explained $75.2 \%$ of the variation in shape, and that association between size and shape was highly significant (Goodall's $F=88.0$, $P<0.001$ ). Thus, the ontogenetic sequence of shapes can be fit to a linear trajectory, with slightly $<25 \%$ of the shape variation left unexplained. Figure 4 shows scores on that trajectory plotted against centroid size. These scores tend to be below the line between $\mathrm{p} 5$ and $\mathrm{p} 10$ and above the line at other ages, suggesting a slight lag in the amount of shape change between $\mathrm{p} 5$ and $\mathrm{p} 10$. An alternative explanation, investigated below, is that these age-related deviations reflect a non-linear trajectory of shape change.

The linear pattern of ontogenetic shape change inferred by regression is shown in Fig. 5 . The most striking feature is the dramatic expansion of the posterior processes relative to the portions of horizontal ramus enveloping the molars and the anterior part of the incisor. The coronoid process tends to become relatively taller with age, while its base becomes broader, giving its anterior edge a more S-shape profile. The condylar processes tends to elongate more than they widen, with the ventral portion extending relatively further posteriorly than the dorsal portion, rotating the condyle to a more horizontal orientation. The angular process expands ventrally much more than it elongates, and at the same time overlaps relatively more of the posterior ramus and incisor. The bone between the processes does not grow posteriorly as fast as the processes do, so the two notches separating them become progressively deeper. Although the base of the coronoid shifts posteriorly from the back of the first molar to the back of the second molar, 
Fig. 3 Fit of monomolecular growth model to data for size (A) and shape (B). Values of asymptotes estimated from the model are indicated by heavy solid lines, $95 \%$ and $90 \%$ of asymptote are indicated by dashed lines. Arrows indicate approximate ages of developmental milestones: $T$, beginning of tooth eruption for $m 1$ (this study); $E$, eye opening (Gao et al. 2002; Sale et al. 2004); W, end of weaning (König \& Markl, 1987; Bechard \& Mason, 2010); R, earliest age of first reproduction or dispersal of wild Mus (Gerlach, 1996; Krackow, 2005).
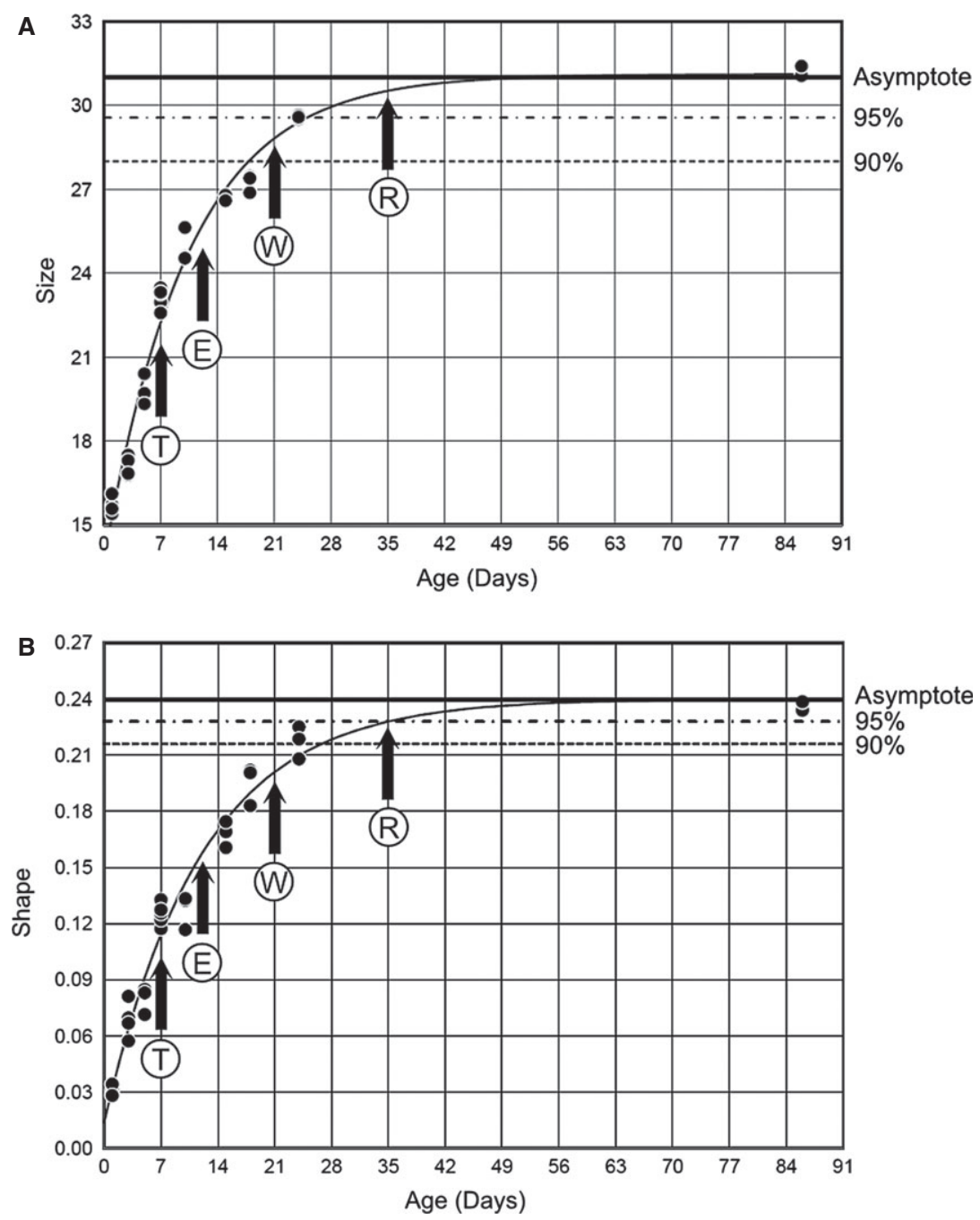
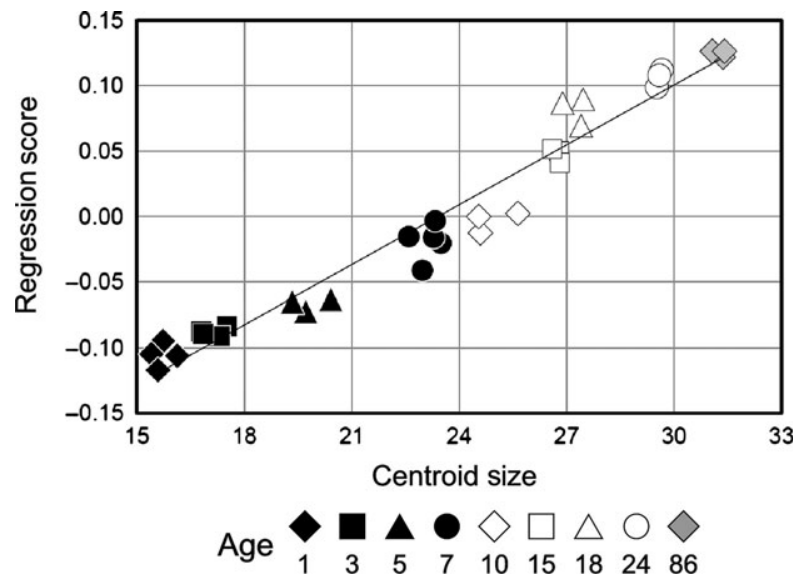

Fig. 4 Relationship between size and shape shown as scatter plots of shape scores against centroid size. Symbols indicate postnatal age in days.

this increasing distance is small relative to the expansion of the posterior processes; thus, the molar region becomes relatively shorter. The entire region to the anterior of the

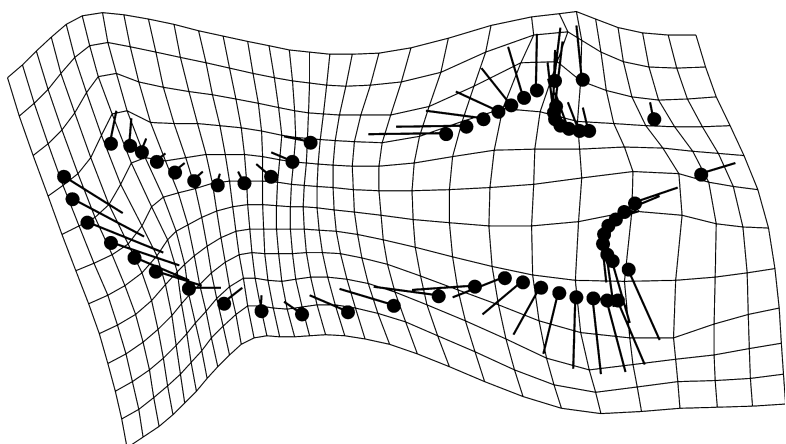

Fig. 5 Pattern of shape change correlated with increasing size, shown as vectors and deformation grid interpolation computed from the thin plate spline.

coronoid and angular processes also becomes relatively deeper, accommodating the elongation of the molars (first the crown, then the roots) and the widening of the incisor. The region below the diastema also elongates but less than it thickens and increases curvature to match the thicker and more curved incisor. 


\section{Trajectory of shape change}

The pattern of shape change described above represents the mean ontogenetic vector, the direction of change that leads from the neonatal shape to the adult shape. Yet to be determined is whether this vector represents the actual ontogenetic path, the direction of change from age to age. If the path is truly linear, then the remaining $25 \%$ of shape variation must represent deviations that are random with respect to age or size. The alternative model is that jaw shape follows a longer, non-linear path, such that the vector described above is the net result - a linearization connecting start to end but not representing all the intervening stages. In that case, much of the $25 \%$ of shape variation that is not described by the net ontogenetic vector may be attributable to age-related changes in the direction of the jaw shape ontogeny.

As the first step to investigate this possibility, we performed PCA on the jaw shape variables. We examined scores for all PCs accounting for more than $1 \%$ of the variation in shape in this sample, to identify intervals in which there might be changes in the direction of the ontogenetic trajectory. This set encompassed the first five PCs and accounts for $95.4 \%$ of the variation.

Scores for PC1 $(78.9 \%$ of variation) and PC2 (10.0\%) clearly define a non-linear trajectory with a sharp change in direction around p7 (Fig. 6). Scores for PC3 (3.1\%) indicate additional deviations from linearity producing shapes above (p3, adult) and below (p1, p18) the PC1-PC2 plane. Scores on PC4 $(2.2 \%)$ and PC5 $(1.3 \%)$ did not make clear contributions to shape differences between ages in any interval.

The direction of shape change described by PC1 (Fig. 7A) differs only slightly from the ontogenetic vector inferred by regression. $\mathrm{PC} 2$ describes the direction accounting for most of the deviation from PC1. At p7, this deviation includes a less narrow ramus and less tilted incisor alveolus aperture, a shorter and broader coronoid process and a more hooked angular process (Fig. 7B). PC3 primarily represents deviations in the shape of the condylar process, which is relatively long and narrow at $\mathrm{p} 3$ in adults, and relatively short and wide at $\mathrm{p} 1$ and $\mathrm{p} 18$ (Fig. 7C).

In light of the PC scores and the developmental milestones described above, we infer there are two major changes in the direction of the ontogenetic trajectory. The first occurs around p7, shortly before tooth eruption begins. The second occurs around p15 at about the beginning of weaning, when $\mathrm{m} 1$ and $\mathrm{m} 2$ have reached the occlusal plane and animals begin forceful chewing. We divided the ontogeny into three intervals demarcated at p7 and p15, described the vector of ontogenetic change within each interval, and tested whether those directions are significantly different.

The directions of change in the first two intervals, before and after p7, differ by more than $60^{\circ}$ (Table 2 ). The cosine
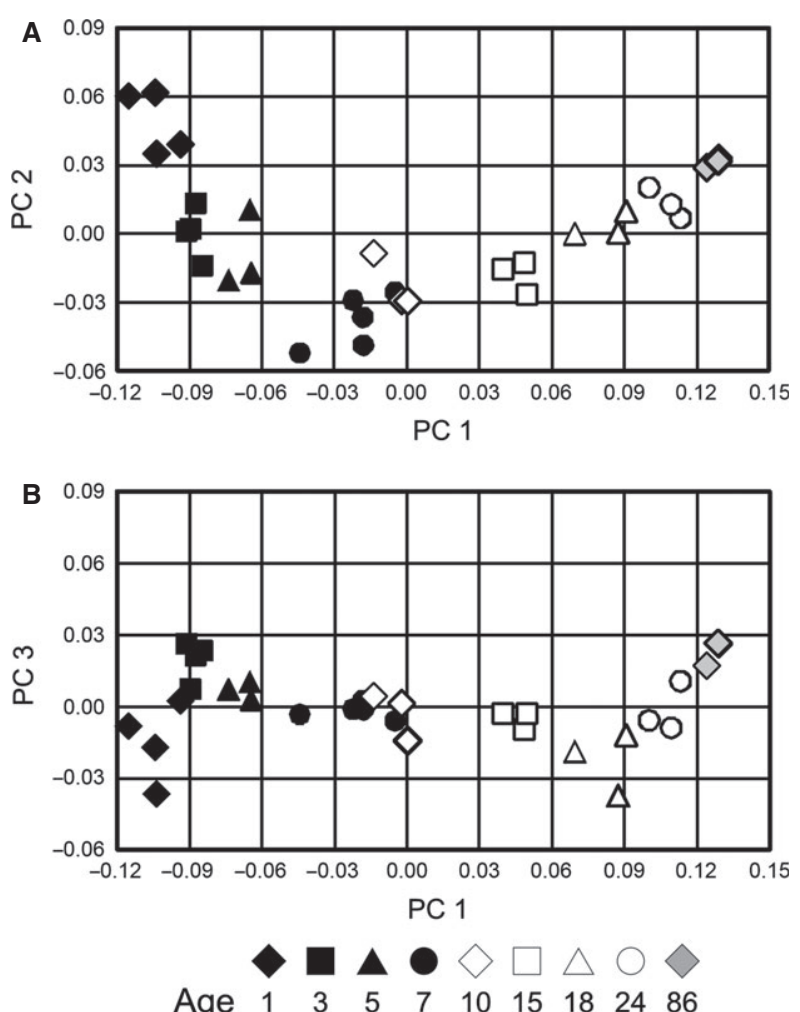

Fig. 6 Scatter plots of PC scores. (A) PC1 1 PC2, (B) PC1 $1 \times P C 3$ Symbols indicate postnatal age in days.

of that angle is about 0.4, suggesting the differences between the trajectories are greater than their similarities. Bootstrapping all the samples in each interval confirms that the angle between them is statistically significant (greater than the confidence interval around either vector). The angle between the second and third intervals is smaller, but still $>45^{\circ}$, and still significant. This second change in direction is not simply a reversal of the first change in direction; such a reversal would produce an angle of about $20^{\circ}$ between the first vector and third. The second change in direction also is not a continuation of the first change in direction; that would produce an angle of about $115^{\circ}$ between the first vector and third. The observed angle of almost $75^{\circ}$ between the first and third intervals is only possible if the third interval is not in the plane defined by the first two. Thus, in each interval, jaw shape moves closer to the adult shape but does not follow a direct line toward the target until the last interval.

The direction of shape change in each interval is shown in Fig. 8. In all three intervals, there is expansion of the three posterior processes relative to the molar alveolar area, while the incisor alveolus and diastema become relatively shorter, broader and more tightly curved. Beyond these broad similarities, however, there are substantial differences between the trajectories within each interval. The relative magnitudes of change among the processes change from one interval to the next; for example, the coronoid changes 
A

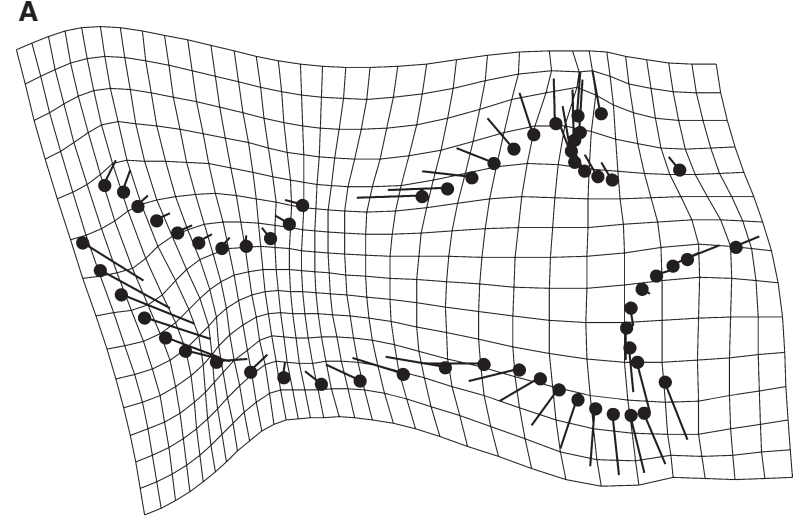

B

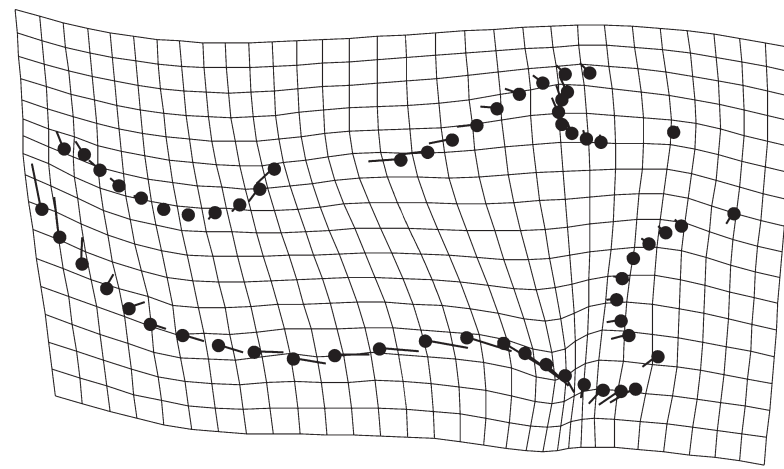

C

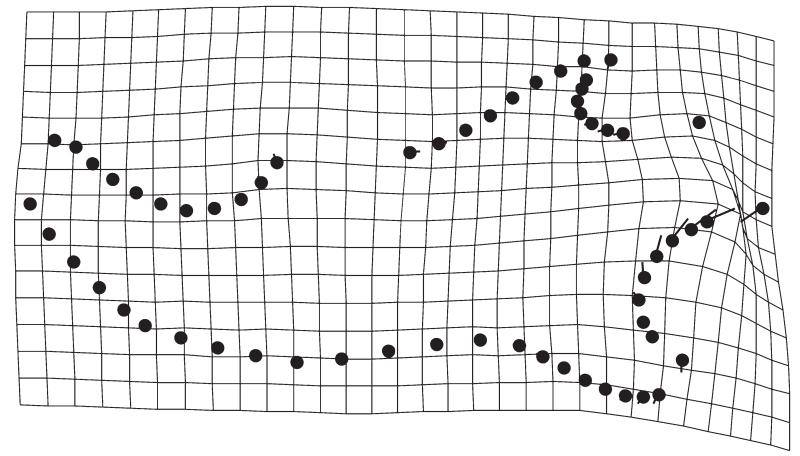

Fig. 7 Patterns of shape change represented by PC scores in the direction of change during the indicated interval: (A) PC1, p1 - adult; (B) PC2, p1 - p7; (C) PC3, p18 - adult. Magnitudes are scaled to reflect relative contributions to net shape change in the indicated interval, except for PC4, which is magnified by a factor of 2 to enhance visibility.

relatively more in the first week, whereas the angular predominates in the last interval. More interesting, the direction of shape change in each region changes from one interval to the next. In the first interval, the diastema becomes narrower, longer and more curved; in the second interval, narrowing is relatively more important and the aperture starts to incline back; in the third interval, the aperture continues to tilt while increasing curvature becomes more prominent than lengthening and the posterior diastema thickens. The angular becomes thicker, shifts posteriorly and becomes more inclined relative to the
Table 2 Analysis of angles between directions of ontogenetic change.

\begin{tabular}{llll}
\hline Age intervals & Angle between intervals & \multicolumn{2}{c}{$\begin{array}{c}\text { Angles within } \\
\text { intervals }\end{array}$} \\
\hline $1-7,7-15$ & 66.4 & 36.7 & 32.1 \\
$7-15,15-$ ad & 46.6 & 32.1 & 26.9 \\
$1-7,15-$ ad & 73.7 & 36.7 & 26.9 \\
\hline
\end{tabular}

All angles between intervals are significantly different from zero. Angles within intervals represent confidence intervals inferred by bootstrap resampling of residuals for individuals within the interval. Angles between are considered significant if they are greater than the larger of the two angles within.
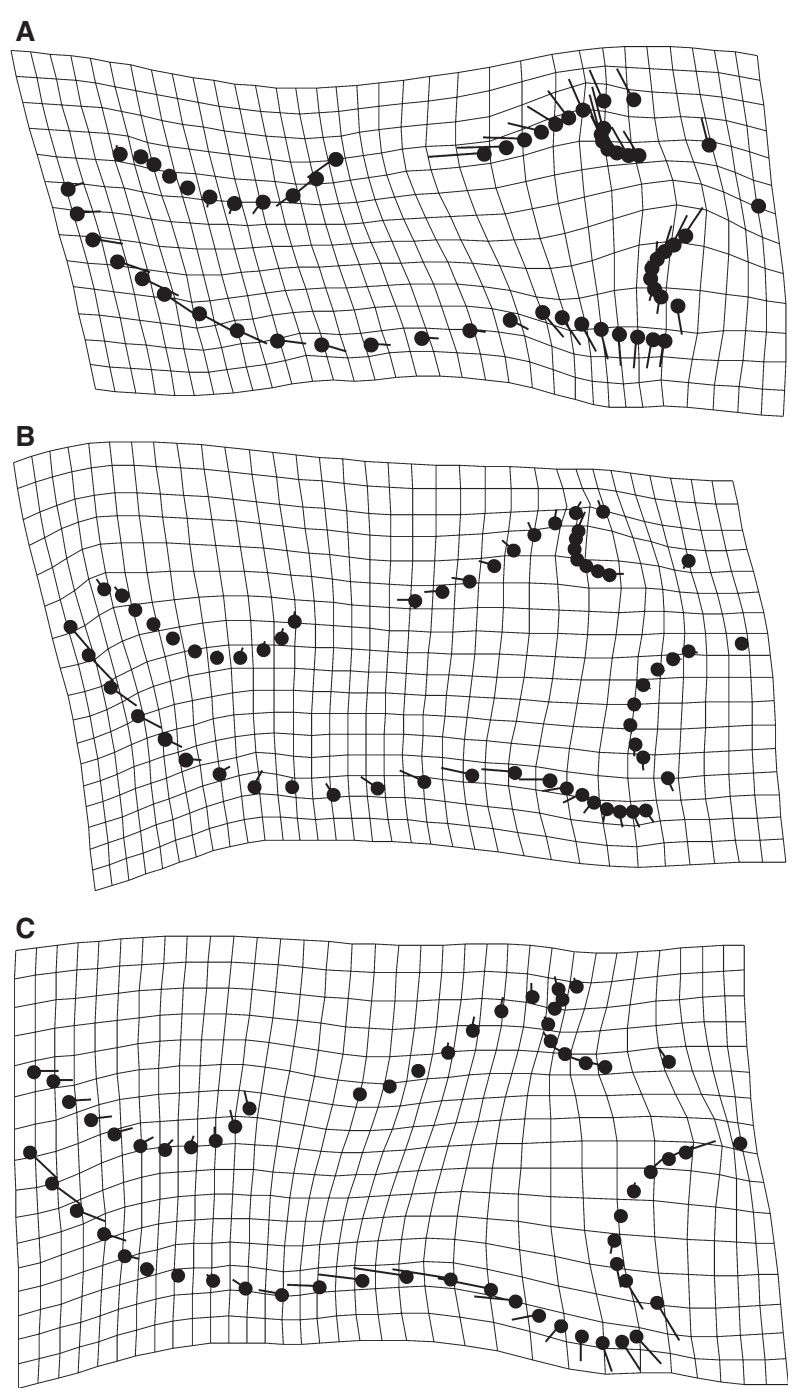

Fig. 8 Net changes in shape over three ontogenetic intervals. (A) p1p7, (B) p7-p15, (C) p15-adult). For each interval, the younger age is starting shape and the older age is the target shape.

horizontal ramus in the first interval; expands relatively more at its base and hooks more at its tip in the second interval; has more uniform thickening and elongation in 
Table 3 Analysis of amount of shape change during 3 postnatal intervals.

\begin{tabular}{lll}
\hline Age intervals & Procrustes distance & Confidence interval \\
\hline $1-7$ & 0.1217 & $0.1101-0.1360$ \\
$7-15$ & 0.0766 & $0.0660-0.0945$ \\
$15-$ ad & 0.1039 & $0.1029-0.1089$ \\
\hline
\end{tabular}

Procrustes distance is the partial Procrustes distance between the means of the samples at each end of the interval. Confidence interval is the range spanned by $95 \%$ of 2500 bootstrap resampling sets.

the third interval. The other regions also have a different direction of shape change in each interval.

One consequence of the changes in direction is that the total path length is considerably longer than the Procrustes distance from $\mathrm{p} 1$ to adult. The lengths of the three intervals (Table 3 ) sum to 0.302 , whereas the net distance from neonate to adult is 0.236 . Thus, the path length is almost $30 \%$ longer than the straight line distance between starting and ending shapes. The differences among the three interval lengths are also notable, with the second and third intervals producing 63 and $85 \%$ as much change as the first. As shown in Table 3, the 95\% confidence intervals for the three lengths do not overlap. In a stronger test for differences in length, the samples for two intervals were simultaneously bootstrapped and the difference in length between each pair of bootstrap sets was computed 2500 times. In these tests, the first interval was always longer than the third, and the third was always longer than the second, supporting the inference that the three lengths are significantly different.

\section{Discussion}

The shape of the mouse mandible follows a complex trajectory from neonate to adult. In the first week, shape change is dominated by deepening of the horizontal ramus and elongation of the condylar process. In the second week, there is greater emphasis on increasing the relative height of the coronoid process, the depth of the angular process and the thickness of condylar process. In the third week and continuing to the adult shape, the prominent features are increasing curvatures of the coronoid and angular processes and especially the diastemal portion of the ramus, along with a marked expansion of the angular and condylar processes. In each interval, there is growth and shape change in every anatomical region, and each change of direction between intervals entails changes in the ontogenetic trajectories of all regions. Thus, the complexity of the overall ontogeny does not stem from simple ontogenies that are expressed sequentially in one region after another, but from complex ontogenies in each region that are unfolding simultaneously.
The small number of ages sampled, especially after p10, and the small numbers of individuals at each age, limit our ability to fit and compare complex models. Nonetheless, it is clear that a linear model of mandibular shape change is a poor fit to the observed distribution of shapes, leaving more than $20 \%$ of the age-related variation unexplained. Although that poor fit, especially the non-random distribution of residuals, suggested a non-linear trajectory, additional information was required to identify the time intervals in which the changes in direction occurred. Using PCA to help visualize the trajectory and the timing of key developmental events, we were able to delineate three intervals with very distinct directions of shape change. What remains to be determined is whether shape change is linear within intervals, and the abruptness of the change in direction between intervals.

One striking feature of the mandibular shape ontogeny of Mus is its close correspondence to the pattern of tooth development. The ventral and anterior parts of the horizontal ramus and condylar process elongate to accommodate the antero-posterior addition of teeth and their expanding crowns. As tooth formation shifts from expanding the crown cross-sectional areas to increasing their heights and then extending their roots, growth of the ramus likewise shifts from elongation (and widening that could not be seen in lateral view) to progressively greater proportions of deepening. By p3, there is little or no dorsoventral separation between alveolar spaces for molars and incisor; consequently, the jaw must deepen at least fast enough to contain both the growing molar crown and the thickening and bowing of the incisor. When molar eruption begins at about p10, their roots have just begun to form; eruption appears to be necessary to make space for growth of the roots. From this age, the rate of bone growth no longer has to match the rate of tooth growth, just the difference between tooth migration upward and root elongation downward. While eruption is nearing completion and active gnawing and chewing begin, changes in ramus shape decrease and changes in shapes of the coronoid and angular processes increase to match the more rapid rate of muscle growth. At the same time, shape change of the condylar process shifts to relatively less elongation and more deepening; it no longer adds to ramus length, but to the robustness needed to withstand higher loads incurred during feeding on solid food.

Other mammals also exhibit complex curving ontogenies of mandibular shape (Cardini \& Tongiorgi, 2003; La Croix et al. 2011; Ventura \& Casado-Cruz, 2011). Analysis of embedded markers (Robinson \& Sarnat, 1955) and histology (Bhaskar, 1953; Enlow \& Harris, 1964; Bang \& Enlow, 1967; de Buffrénil \& Pascal, 1984) suggest such complex ontogenies cannot be completely explained by changes in relative growth of the cartilages but also requires changes in the pattern of periosteal deposition and resorption. Moreover, these studies all report patterns of growth and shape 
change similar to that seen in Mus mandibles. That mammals as diverse as rodents, rabbits, carnivorans, pigs and humans all exhibit similar complex trajectories suggests that this may be a common feature of mandibular development in mammals. One area where differences between taxa might be expected is in the distinctness of the stages and the abruptness of the transitions between stages. Most mammals have more teeth than Mus and relatively later onset of tooth development; longer faces with more teeth and later onset of tooth development could all contribute to smaller angles between directions of shape change and more gradual changes in direction.

Crania, too, exhibit complex curving ontogenies of shape (Hingst-Zaher et al. 2000; Zelditch et al. 2003; Tanner et al. 2010; La Croix et al. 2011). Changes in the direction of cranial shape trajectory may not be as closely tied to tooth development because the cranium encompasses the braincase, sensory capsules and pharynx, and these structures have their own ontogenies. Still, there are aspects of the changes in direction of skull shape ontogeny that reflect the pattern and timing of tooth and muscle development. The principal difference between growth patterns of crania and mandibles is that expansion of the braincase and sensory capsules adds to the complexity of cranial development.

The complex trajectory of mandibular shape might be an adaptation that facilitates juvenile feeding by allowing for a morphology at weaning that is not on a linear trajectory from neonatal to adult shape. Small size and immature shape at weaning puts juveniles at a competitive disadvantage relative to adults (Biknevicius, 1996; Binder \& Van Valkenburgh, 2000; La Croix et al. 2011). However, a morphology that is specifically adapted for juvenile size could allow immature offspring to exploit a transitional diet that reduces competition with the conspecific adults. This strategy has the added benefits that offspring need not be nursed until they are competitive with adults and that, after weaning, any further parental assistance with feeding or foraging can be shared between parents or even with other members of a social group.

The complexity of the mandibular ontogenetic trajectory raises questions about the integration and modularity of the mandible and its development. Numerous studies of phenotypic variation in mandibles of adult mice have been presented as supporting the hypothesis that the mandible is composed of two modules delineated at about the posterior margin of the molar row (Cheverud et al. 1997, 2004; Klingenberg et al. 2003; Burgio et al. 2012; Martinez-Maza et al. 2012). The data collected for this study did not permit explicit testing of this hypothesis, but they are not wholly congruent with it. The changes in direction of the ontogenetic trajectory do not arise because first the anterior region of the jaw grows and changes shape, then the posterior. They also do not arise because the entire anterior region undergoes one change in its ontogenetic trajectory and the entire posterior region undergoes a different change in its trajectory. Instead, there appear to be multiple regions undergoing distinct changes in ontogenetic trajectory simultaneously, with different combinations of changes occurring at the p7 and p15 transitions. Rather than a two-part model, these results might be explained by several small modules undergoing simultaneous changes due to an overarching integration. The many small modules may reflect earlier developmental processes (Atchley \& Hall, 1991); their partial integration may reflect their anatomical merging and functional interdependence (Monteiro et al. 2005; Zelditch et al. 2008, 2009; Monteiro \& Nogueira, 2009). Studies of mandibular modularity have sometimes struggled to explain high levels of covariation between modules (e.g. Klingenberg et al. 2003), and the palimpsest model of sequential, partially overlapping patterns of covariance driven by successive local developmental processes has been proposed to explain how modularity becomes obscured (Hallgrímsson et al. 2009). The results of this study suggest a hierarchical organization of integration could also fit such patterns.

The developmental pattern observed in Mus suggests that growth and form of the posterior processes are never completely independent of the development of the teeth and the horizontal ramus. This non-independence is partly because the condylar process is a continuation of the horizontal ramus - bone is added at the posterior end to provide the length needed to insert progressively more posterior molars. The positions and orientations of the coronoid and angular processes are adjusted to maintain their positions relative to the critical dentition. Increases in the areas and robustness of muscle attachment sites are associated with reinforcement and reshaping of the horizontal ramus to support the increased loads on the teeth and at the mandibular symphysis. Thus, functional interactions of tooth, bone and muscle require coordinated developmental outcomes even if the development of the components is separated in time. The observed pattern of shifting covariances of mandibular regions during postnatal growth is partly consistent with the palimpsest model. However, this pattern differs from that model in that all components are involved in each successive covariance pattern, whereas in the model, different partially overlapping combinations of components are involved in each successive covariance pattern.

Correlated outcomes of temporally disjunct developmental processes also have implications for evolutionary and plastic responses to environmental change. That these responses are larger in the posterior processes than in the horizontal ramus is consistent with the distribution of growth rates after weaning. This difference in magnitudes makes the posterior changes easier to detect; it does not make them independent of more subtle changes occurring in the horizontal ramus. Because tooth development is isolated from the environment and completed much earlier 
than muscle or bone development, the plastic responses of muscle and bone to environmental change will be constrained by the inherited tooth morphology. This dependence of muscle and bone on tooth morphology may explain why lineages with conserved dental morphologies tend to exhibit well defined trajectories of mandibular shape evolution.

The ability to coordinate outcomes of developmental processes separated in time would allow for shifts in timing like the remarkably early onset of molar development seen in Mus. That same ability would also provide a degree of independence between ontogenetic stages that could permit juvenile shapes that are not on a linear trajectory between neonatal and adult shapes. This, in turn, would allow the independent evolution of different stages in continuous ontogeny similar to that afforded by metamorphosis. In addition, the ability to coordinate outcomes of developmental processes that are separated in time would explain plastic or evolutionary changes in one region that do not result in excessive strain in another region, not only permitting the change but also accounting for its limits.

The genetic controls that might provide the overarching integration of multiple developmental modules and define the shape targets for bone growth remain obscure. There have been many analyses of gene activity during formation of the mandible, from migration of neural crest through deposition of the initial osteoid layer enveloping Meckel's cartilage and the tooth germs (Depew et al. 1999; Shibata et al. 2006; Oka et al. 2007; Anthwal et al. 2008; Tsutsui et al. 2008; Zhang et al. 2012). Much less information is available regarding the genetic mechanisms directing subsequent growth. It has long been known that periosteal deposition and resorption contribute to growth and shaping of the ramus and posterior processes throughout this period (Bhaskar, 1953; Robinson \& Sarnat, 1955; Bang \& Enlow, 1967; Ramaesh \& Bard, 2003). Some studies suggest that these changes, as well as outgrowth and the posterior cartilages, can be influenced by hormonal disruptions (Fujita et al. 2004; Ramirez-Yañez et al. 2005), muscle development mutations (Lightfoot \& German, 1998; Nicholson et al. 2006; Ravosa et al. 2007; Rot-Nikcevic et al. 2007; Renaud et al. 2010; Vecchione et al. 2010), changes in food hardness or consistency (He \& Kiliaridis, 2003; Mavropoulos et al. 2005), and orthodontic manipulation (Mavropoulos et al. 2005; Shen et al. 2006). Yet, it remains unclear what regulates the spatial distribution of those signals during normal growth to produce the ontogenetic sequence of shapes (Nicholson et al. 2006; Hsiao et al. 2010; Sun \& Tee, 2011). The results of this study suggest that this spatial distribution is repatterned twice during postnatal growth of the Mus mandible to produce the sharp changes in the direction of its ontogenetic trajectory. We infer that these changes in direction may provide important clues to understanding the patterning of growth as well as we understand its mechanism.
A better understanding of the mechanisms of growth patterning could be instrumental in developing the therapeutic potential for manipulating that growth to correct abnormalities in both size and shape. Humans and other species of interest may not have such sharply delineated changes in the ontogenetic trajectory of shape, but those changes may still be tied to the pattern and timing of tooth development. Thus studies that seek to understand how growth signals are moderated to produce the target shape may benefit from the sharp angles and abrupt directional changes of the Mus ontogeny. The highly angular shape of the Mus ontogeny should help to delineate the phases to be contrasted; subsequent studies can then test whether similar agents produce more subtle changes in the directions of other ontogenies, including ontogenies of bones that do not have teeth.

\section{Acknowledgements}

We thank D. Kohrman and M. Avenarius for generously providing the mice used in this study, and Y. Raphael for support and helpful suggestions. We also thank two anonymous reviewers for their helpful comments. The work was supported by NIH/NIDCD grant P30-DC05188.

\section{References}

Anthwal N, Chai Y, Tucker AS (2008) The role of transforming growth factor- $\beta$ signalling in the patterning of the proximal processes of the murine dentary. Dev Dyn 237, 1604-1613.

Atchley WR, Hall BK (1991) A model for development and evolution of complex morphological structures. Biol Rev 66, 101-157.

Atchley WR, Plummer AA, Riska B (1985) Genetics of mandible form in the mouse. Genetics 111, 555-577.

Bailey DW (1985) Genes that affect the shape of the murine mandible: congenic strain analysis. $J$ Hered 76, 107-114.

Bang S, Enlow DH (1967) Postnatal growth of the rabbit mandible. Arch Oral Biol 12, 993-998.

Bechard A, Mason G (2010) Leaving home: a study of laboratory mouse pup independence. Appl Anim Behav Sci 125, 181-188.

Bhaskar SN (1953) Growth pattern of the rat mandible from 13 days insemination age to 30 days after birth. Am J Anat 92, 1-53.

Biknevicius AR (1996) Functional discrimination in the masticatory apparatus of juvenile and adult cougars (Puma concolor) and spotted hyenas (Crocuta crocuta). Can J Zool 74, 1934-1942.

Binder WJ, Van Valkenburgh B (2000) Development of bite strength and feeding behaviour in juvenile spotted hyenas (Crocuta crocuta). J Zool 252, 273-283.

Boell L, Gregorova S, Forejt J, et al. (2011) A comparative assessment of mandible shape in a consomic strain panel of the house mouse (Mus musculus) - implications for epistasis and evolvability of quantitative traits. BMC Evol Biol 11, 309.

Bookstein FL (1991) Morphometric Tools for Landmark Data: Geometry and Biology. Cambridge: Cambridge University Press.

Bookstein FL (1997a) Landmark methods for forms without landmarks: morphometrics of group differences in outline shape. Med Image Anal 1, 225-243. 
Bookstein FL (1997b) Shape and the information in medical images: a decade of the morphometric synthesis. Comput Vis Image Underst 66, 97-118.

de Buffrénil V, Pascal M (1984) Croissance et morphogénèse postnatales de la mandibule du vison (Mustela vison Schreiber): données sur la dynamique et l'interprétation fonctionnelle des dépôts osseux mandibulaires. Can J Zool 62, 2026-2037.

Burgio G, Baylac M, Heyer E, et al. (2012) Exploration of the genetic organization of morphological modularity on the mouse mandible using a set of interspecific recombinant congenic strains between C57BL/6 and mice of the Mus spretus species. G3 2, 1257-1268.

Cardini A, Tongiorgi P (2003) Yellow-bellied marmots (Marmota flaviventris) 'in the shape space' (Rodentia, Sciuridae): sexual dimorphism, growth and allometry of the mandible. Zoomorphology 122, 11-23.

Cheverud JM, Routman EJ, Irschick DJ (1997) Pleiotropic effects of individual gene loci on mandibular morphology. Evolution 51, 2006-2016.

Cheverud JM, Ehrich TH, Vaughn TT, et al. (2004) Pleiotropic effects on mandibular morphology II: differential epistasis and genetic variation in morphological integration. J Exp Zool $B$ Mol Dev Evol 302B, 424-435.

Depew MJ, Liu JK, Long JE, et al. (1999) DIx5 regulates regional development of the branchial arches and sensory capsules. Development 126, 3831-3846.

Dixon J, Jones NC, Sandell LL, et al. (2006) Tcof1/Treacle is required for neural crest cell formation and proliferation deficiencies that cause craniofacial abnormalities. Proc Natl Acad Sci U S A 103, 13403-13408.

Enlow DH, Harris DB (1964) A study of the postnatal growth of the human mandible. Am J Orthod 50, 25-50.

Fiorello CV, German RZ (1997) Heterochrony within species: craniofacial growth in giant, standard and dwarf rabbits. Evolution 51, 250-261.

Fujita T, Ohtani J, Shigekawa M, et al. (2004) Effects of sex hormone disturbances on craniofacial growth in newborn mice. J Dent Res 83, 250-254.

Gao W-M, Liu H-M, Dong J-C, et al. (2002) Postnatal growth, neurobehavioral and neurophysiologic changes of prenatal low-dose $\beta$-radiation from tritiated water in mice. Neurotoxicol Teratol 24, 247-254.

Gerlach G (1996) Emigration mechanisms in feral house mice - a laboratory investigation of the influence of social structure, population density, and aggression. Behav Ecol Sociobiol 39, 159-170.

Green WDK (1996) The thin-plate spline and images with curving features. In: Proceedings in Image Fusion and Shape Variability Techniques. (eds Mardia KV, Gill CA, Dryden IL), pp. 79-87. Leeds: Leeds University Press.

Hallgrímsson B, Jamniczky H, Young NM, et al. (2009) Deciphering the palimpsest: studying the relationship between morphological integration and phenotypic covariation. Evol Biol 36, 355-376.

He T, Kiliaridis S (2003) Effects of masticatory muscle function on craniofacial morphology in growing ferrets (Mustela putorius furo). Eur J Oral Sci 111, 510-517.

Hingst-Zaher E, Marcus LF, Cerqueira R (2000) Application of geometric morphometrics to the study of postnatal size and shape changes in the skull of Calomys expulsus. Hystrix 11, 99-113.
Hsiao EC, Boudignon BM, Halloran BP, et al. (2010) Gs G protein-coupled receptor signaling in osteoblasts elicits agedependent effects on bone formation. J Bone Miner Res 25, 584-593.

Ishizeki K, Saito H, Shinagawa T, et al. (1999) Histochemical and immunohistochemical analysis of the mechanism of calcification of Meckel's cartilage during mandible development in rodents. J Anat 194, 265-277.

Klingenberg CP, Mebus K, Auffray JC (2003) Developmental integration in a complex morphological structure: how distinct are the modules in the mouse mandible? Evol Dev 5, 522-531.

Klingenberg CP, Leamy $\mathrm{L}$, Cheverud JM (2004) Integration and modularity of quantitative trait locus effects on geometric shape in the mouse mandible. Genetics 166, 1909-1921.

König B, Markl H (1987) Maternal care in house mice. I. The weaning strategy as a means for parental manipulation of offspring quality. Behav Ecol Sociobiol 20, 1-9.

Krackow S (2005) Agonistic onset during development differentiates wild house mouse males (Mus domesticus). Naturwissenschaften 92, 78-81.

La Croix S, Holekamp KE, Shivik JA, et al. (2011) Ontogenetic relationships between cranium and mandible in coyotes and hyenas. J Morphol 272, 662-674.

Lightfoot PS, German RZ (1998) The effects of muscular dystrophy on craniofacial growth in mice: a study of heterochrony and ontogenetic allometry. J Morphol 235, 1-16.

Marcus LF, Corti M, Naylor G, et al., eds (1996) Advances in Morphometrics: Proceedings of the 1993 NATO Advanced Studies Instititute on Morphometrics in II Ciocco, Italy. New York: Plenum Press.

Martinez-Maza C, Montes L, Lamrous H, et al. (2012) Postnatal histomorphogenesis of the mandible in the house mouse. J Anat 220, 472-483.

Mavropoulos A, Ammann P, Bresin A, et al. (2005) Masticatory demands induce region-specific changes in mandibular bone density in growing rats. Angle Orthod 75, 625-630.

McLeod MJ (1980) Differential staining of cartilage and bone in whole mouse fetuses by alcian blue and alizarin red S. Teratology 22, 299-301.

Monteiro LR, Nogueira MR (2009) Adaptive radiations, ecological specialization, and the evolutionary integration of complex morphological structures. Evolution 64, 724-744.

Monteiro LR, Bonato V, dos Reis SF (2005) Evolutionary integration and morphological diversification in complex morphological structures: mandible shape divergence in spiny rats (Rodentia, Echimyidae). Evol Dev 7, 429-439.

Muñoz-Muñoz F, Sans-Fuentes MA, López-Fuster MM, et al. (2011) Evolutionary modularity of the mouse mandible: dissecting the effect of chromosomal reorganizations and isolation by distance in a Robertsonian system of Mus musculus domesticus. J Evol Biol 24, 1763-1776.

Nicholson EK, Stock SR, Hamrick MW, et al. (2006) Biomineralization and adaptive plasticity of the temporomandibular joint in myostatin knockout mice. Arch Oral Biol 51, 37-49.

Oka K, Oka S, Sasaki T, et al. (2007) The role of TGF- $\beta$ signaling in regulating chondrogenesis and osteogenesis during mandibular development. Dev Biol 303, 391-404.

Ramaesh T, Bard JBL (2003) The growth and morphogenesis of the early mouse mandible: a quantitative analysis. J Anat 203, 213-222. 
Ramirez-Yañez GO, Smid JR, Young WG, et al. (2005) Influence of growth hormone on the craniofacial complex of transgenic mice. Eur J Orthod 27, 494-500.

Ravosa MJ, Klopp EB, Pinchoff J, et al. (2007) Plasticity of mandibular biomineralization in myostatin-deficient mice. J Morphol 268, 275-282.

Renaud S, Alibert P, Auffray J-C (2009) Mandible shape in hybrid mice. Naturwissenschaften 96, 1043-1050.

Renaud S, Auffray J-C, de la Porte S (2010) Epigenetic effects on the mouse mandible: common features and discrepancies in remodeling due to muscular dystrophy and response to food consistency. BMC Evol Biol 10, 28.

Robinson IB, Sarnat BG (1955) Growth pattern of the pig mandible - a serial roentgenographic study using metallic implants. Am J Anat 96, 37-63.

Rot-Nikcevic I, Downing KJ, Hall BK, et al. (2007) Development of the mouse mandibles and clavicles in the absence of skeletal myogenesis. Histol Histopathol 22, 51-60.

Ruest LB, Xiang ML, Lim KC, et al. (2004) Endothelin-A receptordependent and -independent signaling pathways in establishing mandibular identity. Development 131, 4413-4423.

Sale A, Putignano E, Cancedda L, et al. (2004) Enriched environment and acceleration of visual system development. Neuropharmacology 47, 649-660.

Sans-Fuentes MA, Ventura J, López-Fuster MJ, et al. (2009) Morphological variation in house mice from the Robertsonian polymorphism area of Barcelona. Biol J Linn Soc 97, 555-570.

Shen G, Zhao Z, Kaluarachchi K, et al. (2006) Expression of type $X$ collagen and capillary endothelium in condylar cartilage during osteogenic transition - a comparison between adaptive remodelling and natural growth. Eur J Orthod 28, 210-216.

Shibata S, Suda N, Suzuki S, et al. (2006) An in situ hybridization study of Runx2, Osterix, and Sox9 at the onset of condylar cartilage formation in fetal mouse mandible. J Anat 208, 169-177.

Siahsarvie R, Auffray JC, Darvish J, et al. (2012) Patterns of morphological evolution in the mandible of the house mouse Mus musculus (Rodentia: Muridae). Biol J Linn Soc 105, 635-647.
Sun Z, Tee BC (2011) Molecular variations related to the regional differences in periosteal growth at the mandibular ramus. Anat Rec 294, 79-87.

Sun Y, Teng I, Huo RD, et al. (2012) Asymmetric requirement of surface epithelial beta-catenin during the upper and lower jaw development. Dev Dyn 241, 663-674.

Tanner JB, Zelditch ML, Lundrigan BL, et al. (2010) Ontogenetic change in skull morphology and mechanical advantage in the spotted hyena (Crocuta crocuta). J Morphol 271, 353-365.

Tsutsui TW, Riminucci M, Holmbeck K, et al. (2008) Development of craniofacial structures in transgenic mice with constitutively active PTH/PTHrP receptor. Bone 42, 321-331.

Vecchione L, Miller J, Byron C, et al. (2010) Age-related changes in craniofacial morphology in GDF-8 (myostatin)-deficient mice. Anat Rec 293, 32-41.

Ventura J, Casado-Cruz M (2011) Post-weaning ontogeny of the mandible in fossorial water voles: ecological and evolutionary implications. Acta Zoologica 92, 12-20.

Zelditch ML, Sheets HD, Fink WL (2000) Spatiotemporal reorganization of growth rates in the evolution of ontogeny. Evolution 54, 1363-1371.

Zelditch ML, Lundrigan BL, Sheets HD, et al. (2003) Do precocial mammals develop at a faster rate? A comparison of rates of skull development in Sigmodon fulviventer and Mus musculus domesticus. J Evol Biol 16, 708-720.

Zelditch ML, Wood AR, Bonett RM, et al. (2008) Modularity of the rodent mandible: integrating bones, muscles, and teeth. Evol Dev 10, 756-768.

Zelditch ML, Wood AR, Swiderski DL (2009) Building developmental integration into functional systems: function-induced integration of mandibular shape. Evol Biol 36, 71-87.

Zelditch ML, Swiderski DL, Sheets HD (2012) Geometric Morphometrics for Biologists: A Primer. 2nd edn. London: Elsevier.

Zhang YP, Blackwell EL, McKnight MT, et al. (2012) Specific inactivation of Twist1 in the mandibular arch neural crest cells affects the development of the ramus and reveals interactions with hand2. Dev Dyn 241, 924-940.

Zullinger EM, Ricklefs RR, Redford KH, et al. (1984) Fitting sigmoidal equations to mammalian growth curves. J Mammal 65, 607-636. 